\title{
VALORES DE MERCADO PARA ATRIBUTOS DE CALÇADOS: UM ESTUDO COM SAPATOS SOCIAIS MASCULINOS NO MERCADO VAREJISTA DA CIDADE DE SÃO PAULO UTILIZANDO O MÉTODO DE PREÇOS HEDÔNICOS
}

\author{
MARKET VALUES FOR ATTRIBUTES OF FOOTWEAR: A STUDY WITH SOCIAL MEN'S SHOES IN \\ THE RETAILER MARKET OF THE CITY OF SÃO PAULO USING THE HEDONIC PRICE METHOD
}

\author{
Alexandre Mendes da Silva \\ Doutor em Administração - FEA-USP. Área de Economia das Organizações. Programa de \\ Varejo - PROVAR. Universidade de São Paulo, Brasil.

\section{Cláudio Felisoni de Angelo} \\ Doutor em Economia- FEA-USP. Área de Economia das Organizações. Docente da Faculdade \\ de Administração, Economia e Contabilidade da Universidade de São Paulo (FEA-USP). Uni- \\ versidade de São Paulo, Brasil.
}

Data de recebimento: 17/07/2017

\section{José Augusto Giesbrecht da Silveira}

Doutor em Economia- FEA-USP. Área de Economia das Organizações. Docente da Faculdade de Administração, Economia e Contabilidade da Universidade de São Paulo (FEA-USP). Universidade de São Paulo, Brasil.

\section{Nuno Manoel Martins Dias Fouto}

Doutor em Economia- FEA-USP. Área de Economia das Organizações. Docente da Faculdade de Administração, Economia e Contabilidade da Universidade de São Paulo (FEA-USP). Universidade de São Paulo, Brasil.

\section{RESUMO}

Esta pesquisa tem como objetivo identificar a relação entre as características dos calçados sociais masculinos e o seu preço de varejo no município de São Paulo. Para isso, o trabalho utiliza o método de preços hedônicos para a identificação e precificação dos atributos mais importantes para o consumidor final. O banco de dados foi composto por 1.120 observações envolvendo lojas localizadas no município de São Paulo. O modelo linear obtido tem poder de explicação para o modelo de 80\%. Os resultados indicam que a variável que mais impacta o preço do sapato social no município de São Paulo é o Couro em Cromo Alemão no cabedal do calçado. Por outro lado, a variável que mais desconta o preço é a presença do Solado de Borracha. A pesquisa pode ajudar fabricantes a desenvolverem produtos com características mais desejáveis aos consumidores e também pode ajudá-los a aperfeiçoar o portfólio de produtos existentes.

Palavras-chave: Análise de regressão múltipla. Calçados sociais masculinos. Modelo de preços hedônicos. Critério Linktest.

\section{ABSTRACT}

This research aims to identify the relationship between the characteristics of men's formal footwear and its retail price in the city o São Paulo. To achieve this goal, the work uses the hedonic price method for the identification and pricing of the most important attributes for the final consumer. The database consistes of 1,120 observations involving stores located in the city of São Paulo. The linear model obtained has an explanatory power of $80 \%$ for the model. The results also indicate that the variable that most impacts the price of the business shoes in São Paulo is the Leather German Chrome in the shoe uppers. On the other hand, the variable that most discount in the price is the presence of the Rubber Soles. The research can help manufacturers develop products with most desirable features to consumers and can help them refine the existing product portfolio too.

Keywords: Multiple regression analysis. Male social footwear. Hedonic price model. Linktest criterion.

Cláudio Felisoni de Angelo cfa@usp.br
José Augusto Giesbrecht da Silveira jags@usp.br
Nuno Manoel Martins Dias Fouto nfouto@usp.br 


\section{INTRODUÇÃO}

O Brasil tem representado um importante papel no mercado calçadista mundial nos últimos 40 anos, detendo o terceiro lugar no ranking dos maiores produtores mundiais de calçados, ficando atrás apenas da China e da Índia (ASSINTECAL, 2011; IEMI, 2014 e 2015). Em 2014, o valor gerado pela produção da indústria calçadista brasileira foi de $R \$ 27,8$ bilhões, gerando divisas de US\$1,1 bilhão em exportações que foram destinadas para mais de 25 países, sendo que Paraguai, Angola, Estados Unidos, França e Argentina foram os principais países importadores dos calçados brasileiros (IEMI, 2015).

A capacidade de produção do Brasil é superada apenas pela China e pela Índia, mas existem diferenças em termos de tamanho absoluto. Enquanto a China produziu mais de 11 bilhões de pares de sapatos e a Índia cerca de 2,4 bilhões, o Brasil fabricou apenas 877 milhões, dos quais 123 milhões foram exportados em 2015 (IEMI, 2015). Apesar da boa aceitação do calçado brasileiro no exterior, a indústria nacional precisa ser fortalecida e protegida de concorrentes estrangeiros que também competem no mercado doméstico, em especial dos fabricantes de calçados de baixo custo da China, uma vez que $86 \%$ da produção nacional é direcionada para este mercado.

Devido ao baixo índice de barreiras à entrada de novos concorrentes, este é um setor que apresenta forte concorrência. Além disso, a heterogeneidade entre as indústrias também estimula o aparecimento de empresas especializadas em determinadas etapas do processo produtivo. A indústria calçadista é caracterizada pelo elevado po- tencial de criação de empregos, devido à simplicidade e o caráter artesanal do processo produtivo, que utiliza baixa qualificação e remuneração da mão-de-obra empregada (Viana; Rocha, 2006). Do ponto de vista microeconômico este é um mercado monopolisticamente competitivo, no qual existem muitas firmas disputando os consumidores e tentando obter lealdade com suas marcas através de propaganda e diferenciação de seus produtos (KUMAR; DEODHAR, 2014).

Apenas $22 \%$ dos calçados produzidos no Brasil foram direcionados para o segmento masculino em 2014 (IEMI, 2015). Apesar de o preço ser um importante elemento para as decisões de compra, os consumidores brasileiros do sexo masculino, que usam os calçados sociais no dia-a-dia de trabalho, estão começando a experimentar calçados com base em outros fatores como estilo, conforto, qualidade e marca do fabricante. Com as mudanças no estilo de vida e na moda, aumento de renda, melhor organização do varejo e a presença de produtos estrangeiros, o mercado de calçados sociais masculinos brasileiros encontra-se atualmente em um momento de transição, fazendo com que seja importante para os fabricantes domésticos a formulação de estratégias que mantenham ou aumentem sua participação no mercado nacional. Isso pode ser feito através de uma melhor compreensão das preferências desses consumidores e da importância que eles atribuem às características (intrínsecas e extrínsecas ${ }^{2}$ ) que são encontradas nesses calçados. Com isso tem-se o problema de pesquisa deste estudo que é: Quais são as principais caracte-

\footnotetext{
1 Neste estudo as palavras atributo, características e fatores são utilizados de forma intercambiável.

2 Intrínsecos - que são constitutivos do produto / extrínsecos - que são externos ao produto.
} 
rísticas e seus preços implícitos dos calçados sociais masculinos comercializados no mercado varejista do município de São Paulo?

Esta pergunta nos leva ao objetivo desta pesquisa, que é utilizar o método dos preços hedônicos para determinar empiricamente o valor e a importância relativa dos atributos no preço dos calçados sociais masculinos no varejo da cidade de São Paulo. Este método se caracteriza pela obtenção dos preços implícitos dos atributos de um bem, para os quais não existe um mercado direto, e a partir disso pode-se estabelecer a importância relativa atribuída a cada atributo, ao mesmo tempo obtendo-se o valor atribuído pelos consumidores aos itens que compõem (FOUTO; FRANCISCO 2010). A hipótese deste trabalho é que as características dos calçados sociais masculinos podem ser precificados utilizando o método de preços hedônicos.

Diversas pesquisas já foram desenvolvidas abordando a indústria calçadista nacional, tanto em termos de estratégia (ALVES FILHO, 1991; BIMBATTI, 1994), modernização (COSTA, 1993), mercado (FENSTERSEIFER et al., 1995; CONSTANZI, 1999), competitividade (COSTA, 2004), cadeia produtiva (CARDOSO et al., 2001), exportação (MACHADO NETO, 2006), marcas (GRANERO, 2008). Mais recentemente, foram elaborados estudos que tratam do design (Menezes e Paschoarelli, 2009) e de práticas de gestão ambiental utilizadas pelas indústrias coureiras em Franca (Alves e Barbosa, 2013), entre outros. No exterior a indústria de calçados também foi abordada por diversos estudos, como por exemplo, o de Verdu-Jover, Gomez-Graz e Martinez-Mateo (2012) que abordou a criação de valor através de estratégias offshore e inshore em um cluster calçadista em Alicante (Espanha). Outra pesquisa, fei- ta em Portugal por Meneses, Brito e Gomes (2016) estudou a evolução de fabricantes portugueses de fornecedores terceirizados em fabricantes de marca direta, concorrendo com os seus ex-clientes.

No Brasil ainda não existe um estudo que analise os preços dos calçados, ainda mais dos sociais masculinos, a partir de seus atributos. Por isso, um estudo que trabalhe com o tema aqui proposto tem o potencial de ser uma importante contribuição para esse setor da economia nacional.

Para atingir o objetivo deste estudo, o artigo está estruturado da seguinte forma: na seção 2 é apresentado o método dos preços hedônicos. $\mathrm{Na}$ seção 3 encontram-se a metodologia da pesquisa, os resultados e sua discussão. Por fim na seção 4 têm-se as considerações finais desta pesquisa.

\section{O MÉTODO DE PREÇOS HEDÔNICOS}

Etimologicamente, o termo hedônico é derivado da palavra grega hedonikos, que significa prazer. Em economia significa utilidade ou satisfação que deriva do consumo de bens e serviços (Chau; Chin, 2003). A ideia do método dos preços hedônicos é de que o preço de um bem está relacionado com a quantidade de características que o mesmo possui. E a ferramenta que é utilizada para se encontrar os preços implícitos associados atributos a partir dos preços observados dos produtos diferenciados (Rosen, 1974) é a regressão linear múltipla.

Em termos de fundamentação teórica, duas abordagens contribuíram para o método dos preços hedônicos. A primeira é derivada da teoria do consumidor de Lancaster (1966) e a segunda 
é oriunda do modelo postulado por Rosen (1974). Ambas as abordagens visam precificar as características de um bem baseada na relação entre os preços observados de produtos diferenciados e o número de características associadas com esses produtos (CHAU; CHIN, 2003).

Os modelos de Lancaster (1966), Rosen (1974) e o de preços hedônicos pressupõem que os bens possuem um leque de atributos que se combinam para formar pacotes de características (ou objetivamente, utilidade que afeta as características) que o consumidor precifica. Mas esses modelos possuem diferenças importantes. O modelo de Lancaster pressupõe que os bens são membros de um grupo, e que alguns desses bens são consumidos em combinações que são sujeitas às restrições orçamentárias do consumidor. O modelo de Rosen, por sua vez, assume que existe variedade de bens, porém o consumidor não adquire as características preferidas comprando uma combinação de bens. Em vez disso, cada bem é escolhido de um espectro de marcas e consumido discretamente. A abordagem de preços hedônicos também não exige o consumo em conjunto de bens. A abordagem de Lancaster é considerada mais adequada para bens de consumo, enquanto que a de Rosen é mais apropriada a bens de consumo duráveis (Chau; Chin, 2003). Como sapatos são considerados bens de consumo semiduráveis, o Modelo de Rosen (1974) foi adotado neste trabalho.

Para este autor, o método de preços hedônicos assume que bens de mercado consistem em um conjunto de características ( $Z$ ) que podem ser representadas por um vetor $k$ de atributo:

$$
Z=\left(Z_{1}, Z_{2}, \ldots, Z_{k}\right)(\text { Equação } 1)
$$

Sob essa premissa, a função utilidade (U) para o consumidor é expressa como

$$
U=U\left(Z_{1}, Z_{2}, \ldots, Z_{k} ; \alpha\right)(\text { Equação } 2)
$$

Onde $Z_{k}$ é a quantidade $k$ de atributos que estão em um determinado bem e $\alpha$ é o parâmetro das preferências dos consumidores. O nível do k-ésimo atributo obtido pelo consumidor irá depender do número de unidades $\left(Q_{i}\right)$ dos diferentes bens de mercado que estão sendo consumidos. As unidades são relacionadas $a Z_{k}$ através da variável $X_{j k}$, a qual representa o total dos atributos $k$ contidos em uma unidade do j-ésimo produto. Baseado nisso, é possível escrever z como

$$
Z_{k}=f_{k}\left(Q_{1}, Q_{2^{\prime}} \ldots, Q_{n^{\prime}} X_{1 k^{\prime}} X_{2 k^{\prime}} \ldots, X_{n k}\right)(\text { Equação } 3 \text { ) }
$$

Integrando as equações 2 e 3, um nível de utilidade individual é baseada no nível de atributo por unidade do produto e no número de produtos consumidos (Equação 4):

$$
U=U\left(Q_{1}, Q_{2^{\prime}}, \ldots, Q_{n^{\prime}} X 1_{k^{\prime}} X 2_{k^{\prime}} \ldots, X_{n k}\right)(\text { Equação } 4)
$$

De acordo com a teoria econômica, o consumidor irá maximizar a equação 4 sujeita às restrições orçamentárias (M) definidas como

$$
M=(\text { Equação } 5 \text { ) }
$$

Onde Pj representa o preço do $j_{\text {th }}$ bem de mercado. A solução para a maximização deste problema é:

$$
\left.P_{j}=f X_{j 1} ; X_{j 2} ; \ldots ; X_{j k}\right)(\text { Equação 6) }
$$


Onde $X_{j k}$ é a quantidade de atributos $k$ associados a uma unidade de $Q_{j}$. Na equação $6 P$ é preço de um bem e $X$ é o vetor das características de cada bem. Esta função é a equação de regressão de preços hedônicos, a qual é obtida como resultado da comparação de preços de bens heterogêneos.

A equação 6 estabelece que existe uma relação entre o preço e as características do calçado social masculino. O objetivo da estimação de preços hedônicos é determinar o modelo que possa estabelecer essa relação funcional da melhor maneira. De acordo com a literatura (Brachinger, 2003; Kempf, 2015), o modelo de preços hedônicos costuma utilizar quatro formas funcionais paramétricas que podem ser visualizadas no Quadro 1:

Quadro 1 - Formas funcionais empregadas no modelo de preços hedônicos

\begin{tabular}{|c|c|}
\hline Nome do modelo & Equação do modelo \\
\hline Modelo linear (Lin-Lin) & $P=\alpha+\beta_{1} X 1+\beta 2 X 2+\ldots+\beta n X n+\varepsilon$ \\
\hline Modelo duplo logarítmico (Log-Log) & $\operatorname{Ln}(P)=\alpha+\beta 1 \ln (X 1)+\beta 2 \ln (X 2)+\ldots+\beta n \ln (X n)+\varepsilon$ \\
\hline Modelo Linear-Logarítmico (Lin-Log) & $P=\alpha+\beta 1 \ln (X 1)+\beta 2 \ln (X 2)+\ldots+\beta n \ln (X n)+\varepsilon$ \\
\hline Modelo Logarítmico - Linear (Log-Lin) & $\operatorname{Ln}(P)=\alpha+\beta 1 X 1+\beta 2 X 2+\ldots+B n X n+\varepsilon$ \\
\hline
\end{tabular}

Fonte: Gujarati (2006)

Os modelos hedônicos são sensíveis na escolha da forma funcional, porém a teoria econômica não fornece diretrizes claras de como selecionar a melhor forma funcional (Xiao, 2016). Rosen (1974) demonstrou que a forma funcional do preço hedônico é uma equação reduzida que reflete simultaneamente os mecanismos de oferta e de demanda. Em equilíbrio, o valor de qualquer bem econômico é baseada em sua utilidade com base em seus atributos, e nesse contexto as funções de demanda e de oferta são tangentes e a função de preços hedônicos é o envoltório superior para a função de demanda (Figura 1).

O método de preços hedônicos já foi utilizado nos mais diversos tipos de estudo como em imóveis (Baggio, Catapan e Meza, 2015), automóveis (Griliches, 1961; Triplett, 1969), cereais de café da manhã (Stanley; Tschirhart, 1991), ovos (Karipidis et al., 2005), carne (Dutton et al, 2007), peixe congelado (Roheim et al., 2007), vinhos (Orrego; Defrancesco; Gennari, 2012), computadores pessoais
Figura 1 - Função de equilíbrio de preços hedônicos e atributos de um bem

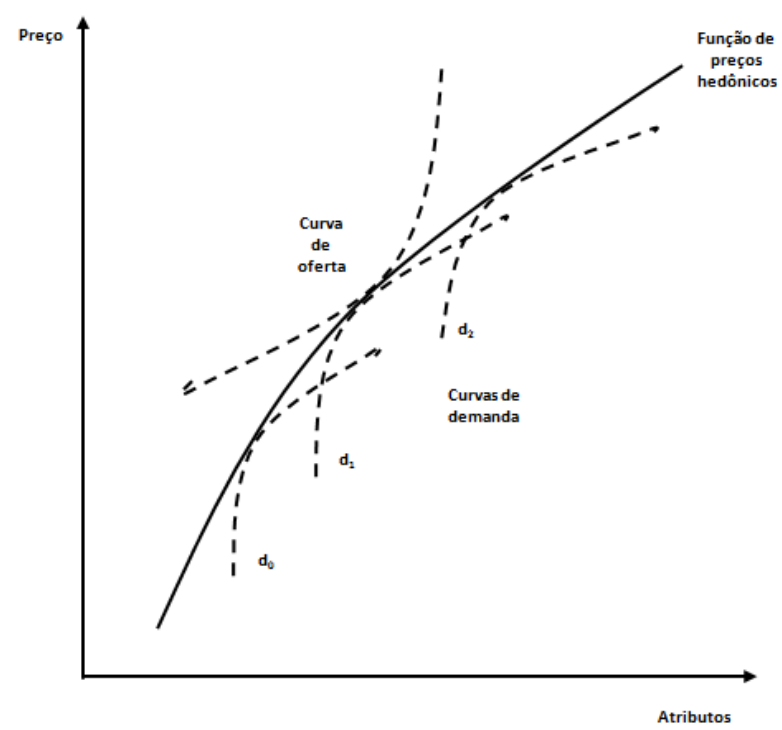

Fonte: Adaptado de Aguirre e Faria (1997, p. 394)

(Fouto; Angelo; Luppe, 2009), produtos de áudio como Compact Disk (CD) players portáteis, receivers, rádios portáteis, entre outros (Kokoski; Waehrer; Rozaklis, 2000), precificação de terras agrícolas com e sem erosão (Campos; Cirino; Andrade, 2004), qualidade do ar e crime no Chile (Dresdner; Lavin; Agui- 
lar, 2011), azeite de oliva no Chile (Muñoz; Moya; Gil, 2015), em transações envolvendo animais como ovelhas e cabras na África (Jabbar, 1998) e em dotes de casamento em função dos atributos sócio-econômicos e demográficos de noivas e noivos em aldeias no Sul da Índia (RAO, 1993).

Mais recentemente o método dos preços hedônicos foi utilizado para avaliar a acessibilidade ao mercado e os preços de hotéis no Caribe (Yang; Mueller; Croes, 2016). Em outro estudo, também foi utilizado para avaliar a relação entre a poluição do ar e a cobertura florestal e o preço das residências no condado de Salt Lake, em Utah - Estados Unidos da América (Li et al., 2016). Na pesquisa de Buonanno, Montolioc e Raya-Vilchez (2013) método dos preços hedônicos foi utilizado para avaliar o efeito da percepção da criminalidade na precificação das moradias na cidade de Barcelona, entre 2004 e 2006.

Pradier et al. (2016) estudaram o mercado de autógrafos no período de 1960 a 2006. Os resultados obtidos com a estimação dos preços hedônicos mostrou que a contagem de páginas, tipo de autor, data e tipo de documento explicam mais de
$75 \%$ das diferenças de preços no mercado. Em calçados esse tipo de análise foi aplicado pela primeira vez por Kumar e Deodhar (2014), que realizaram um estudo no mercado indiano com os calçados sociais masculinos visando identificar e valorar as características preferidas pelos consumidores no mercado indiano. Para isso os autores, após coletarem uma amostra de 150 observações de sapatos de 18 marcas concluíram que em um mercado competitivo, estudos como o que foi realizado podem ajudar as firmas a alterarem e lançarem com sucesso novos modelos de calçados.

\section{PROCEDIMENTOS METODOLÓGICOS}

A amostragem realizada para este estudo foi obtida por conveniência, e envolveu visitas físicas em 21 lojas físicas no município de São Paulo, bem como consulta aos respectivos sites de cada loja na internet. Os dados foram coletados entre os meses de junho e novembro de 2015 e totalizaram 1.120 observações e envolveram 21 lojas e redes de lojas (Quadro 2).

Quadro 2 - Redes e lojas individuais onde foram levantadas as informações da pesquisa

\begin{tabular}{|c|c|}
\hline Louie São Paulo & Companhia Nacional de Sapatos (CNS) \\
\hline Pacco Sapatos & Sapataria Cometa \\
\hline Shoestock & Binne Comfort \\
\hline Mundial Calçados & Di Pollini \\
\hline Calçados Manuel & Pontal Calçados \\
\hline Sapatos Birello & Milano Calçados \\
\hline Porto Free & Gabriella Calçados \\
\hline Lojas Marcom & Alex Shoes \\
\hline Comércio de Calçados Mônica & Fascar \\
\hline Casa Eurico & Calçados Pixolé \\
\hline Atenas Calçados & \\
\hline
\end{tabular}

Fonte: Silva (2017) 
Dessas lojas, $11(52,4 \%)$ estavam situadas na rua (Quadro 3) e 10 em lojas (47,6\%) estabelecidas em shopping-centers (Quadro 4). Em todas as visitas foram levantados os preços e as características dos sapatos (em média 25 levantamentos por visita, envolvendo interação com os vendedores e observação dos calçados expostos na vitrine).

Quadro 3- Relação de empresas visitadas com estabelecimentos situados na rua

\begin{tabular}{|c|c|}
\hline Nome da loja & Endereço \\
\hline Louie São Paulo & Rua Helena \\
\hline Pacco Sapatos & Rua Clodomiro Amazonas, 248 \\
\hline Shoestock & Av. Bem Te Vi, 221 \\
\hline Mundial Calçados & Rua 25 de Março, 831 \\
\hline Calçados Manuel & Rua Major Sertório, 438 \\
\hline Sapatos Birello & Rua Guaicurus, 82 \\
\hline Porto Free & Rua Maria Antônia, 185 \\
\hline Lojas Marcom & Av. Carlos Lacerda, 1804 \\
\hline Comércio de Calçados Mônica & Av. Jardim Japão, 1214 \\
\hline Casa Eurico & Av. Jandira, 49 \\
\hline Atenas Calçados & Rua Domingos de Morais, 413 \\
\hline
\end{tabular}

Fonte: Silva (2017)

Quadro 4 - Relação de lojas visitadas com estabelecimentos situados em shopping-centers

\begin{tabular}{|c|c|}
\hline Nome da loja & Shopping \\
\hline Companhia Nacional de Sapatos (CNS) & Bourbon \\
\hline Sapataria Cometa & Iguatemi \\
\hline Binne Comfort & Morumbi \\
\hline Di Pollini & Eldorado \\
\hline Pontal Calçados & Aricanduva \\
\hline Milano Calçados & Center Norte \\
\hline Gabriella Calçados & Tietê Plaza Shopping \\
\hline Alex Shoes & Metrô Tatuapé \\
\hline Fascar & Villa Lobos \\
\hline Calcados Pixolé & Anália Franco \\
\hline
\end{tabular}

Fonte: Silva (2017)

Mesmo sendo a amostragem intencional, houve a preocupação de se visitar lojas que estivessem situadas nas diferentes regiões da cidade. Das lojas visitadas para a composição da amostra, 5 $(23,8 \%)$ foram na Zona Oeste (Sapatos Birello, Companhia Nacional de Sapatos (CNS), Sapataria Cometa, Di Pollini e Fascar); 3 (14,3\%) foram na Zona Norte (Comércio de Calçados Mônica, Gabriella Cal- çados e Milano Calçados); na Zona Sul foram visitadas 7 lojas (33,3\%) (Louie São Paulo, Pacco Sapatos, Shoestock, Lojas Marcom, Casa Eurico, Atenas Calçados e Binne Comfort); na Zona Leste foram visitadas 3 lojas (14,3\%) (Pontal Calçados, Alex Shoes e Calçados Pixolé); e na Zona Central, por sua vez, foram $3(14,3 \%)$ as lojas visitadas (Mundial Calçados, Calçados Manuel e Porto Free) (Quadro 5). 
Quadro 5- Localização das lojas visitadas por região da cidade de São Paulo

\begin{tabular}{|c|c|}
\hline Lojas & Zona \\
\hline Sapatos Birello & \multirow{5}{*}{ Oeste } \\
\hline Companhia Nacional de Sapatos (CNS) & \\
\hline Sapataria Cometa & \\
\hline Di Pollini & \\
\hline Fascar & \\
\hline Comércio de calçados Mónica & \multirow{3}{*}{ Norte } \\
\hline Gabriella Calçados & \\
\hline Milano Calçados & \\
\hline Louie São Paulo & \multirow{7}{*}{ Sul } \\
\hline Pacco Sapatos & \\
\hline Shoestock & \\
\hline Lojas Marcom & \\
\hline Casa Eurico & \\
\hline Atenas Calçados & \\
\hline Bine Comfort & \\
\hline Pontal & \multirow{3}{*}{ Leste } \\
\hline Alex Shoes & \\
\hline Calçados Pixolé & \\
\hline Mundial Calçados & \multirow{3}{*}{ Central } \\
\hline Calçados Manuel & \\
\hline Porto Free & \\
\hline
\end{tabular}

Fonte: Silva (2017)

Mas apenas as visitas não eram suficientes para a elaboração do banco de dados necessário para esta pesquisa. Por isso, também foram consultados os sites dessas empresas para a coleta de informações sobre os calçados, sendo este o mesmo procedimento utilizado por $\mathrm{Ku}$ mar e Deodhar (2014) em sua pesquisa sobre sapatos sociais masculinos na Índia. O número de observações coletadas por loja pode ser visualizado no Tabela 1:

Tabela 1 - Redes e lojas individuais onde foram levantadas as informações da pesquisa

\begin{tabular}{lllll}
\multicolumn{1}{c}{ Louie São Paulo } & $\mathbf{2 7}$ & \multicolumn{2}{c}{$\begin{array}{c}\text { Companhia Nacional de } \\
\text { Sapatos (CNS) }\end{array}$} & $\mathbf{6 4}$ \\
\hline Pacco Sapatos & 21 & Sapataria Cometa & 8 \\
\hline Shoestock & 27 & Binne Comfort & 11 \\
\hline Mundial Calçados & 39 & Di Pollini & 47 \\
\hline Calçados Manuel & 109 & Pontal Calçados & 26 \\
\hline Sapatos Birello & 29 & Milano Calçados & 30 \\
\hline Porto Free & 64 & Gabriella Calçados & 35 \\
\hline Lojas Marcom & 32 & Alex Shoes & 54 \\
\hline
\end{tabular}




\begin{tabular}{llll}
\hline $\begin{array}{l}\text { Comércio de Calçados } \\
\text { Mônica }\end{array}$ & 70 & Fascar & 169 \\
\hline Casa Eurico & 129 & Calçados Pixolé & 119 \\
\hline Atenas Calçados & 10 & & \\
\hline
\end{tabular}

Fonte: Silva (2017)

O maior preço encontrado foi de

$\mathrm{R} \$ 1.000,00 /$ par e o menor de $\mathrm{R} \$ 50,00 /$ par. O pre-

ço médio foi de $R \$ 248,41 /$ par.

O conjunto de atributos que consti-

tuem as variáveis independentes utilizadas neste estudo totalizou 18 variáveis independentes e uma dependente (o preço de varejo do calçado). No Quadro 6 estão listadas as variáveis independentes.

Quadro 6- Variáveis independentes e seus níveis

\begin{tabular}{|c|c|c|c|}
\hline Nome da variável & $\begin{array}{c}\text { Tipo de } \\
\text { característica }\end{array}$ & Código & Níveis \\
\hline Localização da loja & Extrínseca & LOC & $\begin{array}{l}\text { Rua: } L O C=0 \\
\text { Shopping: } L O C=1\end{array}$ \\
\hline Tipo de canal de distribuição & Extrínseca & CAN & $\begin{array}{l}\text { Loja de rede: } \mathrm{CAN}=0 \\
\text { Loja independente: } \mathrm{CAN}=1\end{array}$ \\
\hline Região da cidade de São Paulo & Extrínseca & REG & $\begin{array}{l}\text { Oeste: } \mathrm{REG}_{1}=0, \mathrm{REG}_{2}=0, \mathrm{REG}_{3}=0, \mathrm{REG}_{4}=0 ; \\
\text { Norte: } \mathrm{REG}_{1}=0, \mathrm{REG}_{2}=0, \mathrm{REG}_{3}=0, \mathrm{REG}_{4}=1 ; \\
\text { Sul: } \mathrm{REG}_{1}=0, \mathrm{REG}_{2}=0, \mathrm{REG}_{3}=1, \mathrm{REG}_{4}=0 ; \\
\text { Leste: } \mathrm{REG}_{1}=0, \mathrm{REG}_{2}=1, \mathrm{REG}_{3}=0, \mathrm{REG}_{4}=0 ; \\
\text { Central: } \mathrm{REG}_{1}=1, \mathrm{REG}_{2}=0, \mathrm{REG}_{3}=0, \mathrm{REG}_{4}=0\end{array}$ \\
\hline $\begin{array}{l}\text { Classificação dos distritos } \\
\text { municipais }\end{array}$ & Extrínseca & CLAS & $\begin{array}{l}\text { Nobre: } \mathrm{CLAS}=0 \\
\text { Popular: } \mathrm{CLAS}=1\end{array}$ \\
\hline Marca de calçado & Extrínseca & MARCA & $\begin{array}{l}\text { Conhecida: } \text { MARC }=0 \\
\text { Pouco conhecida: } M A R C=1\end{array}$ \\
\hline Número de parcelas & Extrínseca & NPARC & Varíavel discreta \\
\hline $\begin{array}{l}\text { Modelo de calçado social } \\
\text { masculino }\end{array}$ & Intrínseca & MOD & $\begin{array}{l}\text { Oxford: MOD1 =0, MOD2 =0, MOD3 = 0, MOD4 } \\
=0, \text { MOD5 = 0; Monk: MOD1 =0, MOD2 =0, } \\
\text { MOD3 = 0, MOD4 = 0, MOD5 = 1; } \\
\text { Derby: MOD1 =0, MOD2 =0, MOD3 = 0, MOD4 } \\
=1, \text { MOD5 =0; } \\
\text { Loafer: MOD1 =0, MOD2 =0, MOD3 = 1, MOD4 } \\
=0, \text { MOD5 =0; } \\
\text { Side Gore: MOD1 =1, MOD2 =0, MOD3 = } 0, \\
\text { MOD4 = 0, MOD5 =0' }\end{array}$ \\
\hline Agrupamento do tipo de calçado & Extrínseca & AGRUP & $\begin{array}{l}\text { Grupo A (Oxford, Derby e Brogue): AGRUP = } 0 \\
\text { Grupo B(Monk, Loafer e Side Gore): AGRUP = } 1\end{array}$ \\
\hline Origem do calçado & Extrínseca & ORCAL & Sul: ORCAL $=0$; Sudeste: ORCAL $=1$ \\
\hline
\end{tabular}




\begin{tabular}{|c|c|c|c|}
\hline Composição do cabedal & Intrínseca & $\mathrm{COU}$ & 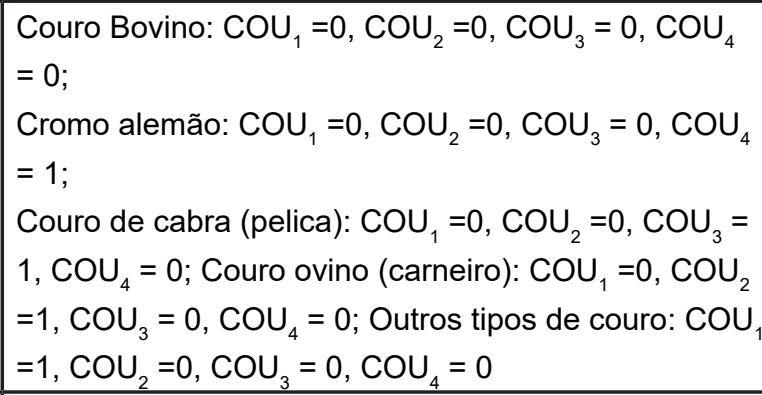 \\
\hline Cor & Intrínseca & COR & $\begin{array}{l}\text { Preto: } \mathrm{COR}_{1}=0, \mathrm{COR}_{2}=0 \\
\text { Marrom: } \mathrm{COR}_{1}=0, \mathrm{COR}_{2}=1 \\
\text { Outras cores: } \mathrm{COR}_{1}=1, \mathrm{COR}_{2}=0\end{array}$ \\
\hline $\begin{array}{l}\text { Acabamento no cabedal - } \\
\text { equivalente a variável Textura } \\
\text { do estudo de Kumar e Deodhar } \\
\text { (2014) }\end{array}$ & Intrínseca & $A C A B$ & $\begin{array}{l}\text { Verniz: } A C A B 1=0, A C A B 2=0, A C A B 3=0, \\
A C A B 4=0, A C A B 5=0 ; \text { Camurça: } A C A B 1=0, \\
A C A B 2=0, A C A B 3=0, A C A B 4=0, A C A B 5=1 ; \\
\text { Estampagem (imitação de couro de cobra, } \\
\text { avestruz, crocodilo, etc.): } A C A B 1=0, A C A B 2=0, \\
\text { ACAB3 = 0, ACAB4 = 1, ACAB5=0; } \\
\text { Nobuck: } A C A B 1=0, A C A B 2=0, A C A B 3=1, \\
\text { ACAB4 =0, ACAB5=0; Liso: ACAB1 =0, ACAB2 } \\
=1, A C A B 3=0, A C A B 4=0, A C A B 5=0 ; \text { Outros: } \\
\text { ACAB1 =1, ACAB2 =0, ACAB3 = 0, ACAB4 =0, } \\
\text { ACAB5 =0 }\end{array}$ \\
\hline Solado & Intrínseca & SOL & $\begin{array}{l}\text { Couro: } \mathrm{SOL}_{1}=0, \mathrm{SOL}_{2}=0 \\
\text { Borracha (natural ou sintética): } \mathrm{SOL}_{1}=0, \mathrm{SOL}_{2}=1 \text {; } \\
\text { Misto (couro + borracha): } \mathrm{SOL}_{1}=1, \mathrm{SOL}_{2}=0 ;\end{array}$ \\
\hline $\begin{array}{l}\text { Bico - equivalente a variável } \\
\text { Estrutura do estudo de Kumar e } \\
\text { Deodhar (2014) }\end{array}$ & Intrínseca & $\mathrm{BIC}$ & $\begin{array}{l}\text { Redondo: } \mathrm{BIC}=0 \\
\text { Quadrado ou afilado: } \mathrm{BIC}=1\end{array}$ \\
\hline Laço & Intrínseca & LAC & $\begin{array}{l}\text { Sim: } L A C=0 \\
\text { Não: } L A C=1\end{array}$ \\
\hline $\begin{array}{l}\text { Acabamento de superfície - } \\
\text { equivalente a variável Superfície } \\
\text { do estudo de Kumar e Deodhar } \\
\text { (2014) }\end{array}$ & Intrínseca & SURF & $\begin{array}{l}\text { Brilhante: SURF1 = 0, SURF2 =0 } \\
\text { Semi-fosco: SURF1 = 0, SURF2 = } 1 \\
\text { Fosco: SURF1 = 1, SURF2 =0 }\end{array}$ \\
\hline Fivela & Intrínseca & FIV & $\begin{array}{l}\text { Sim: } F I V=0 \\
\text { Não: } F I V=1\end{array}$ \\
\hline $\begin{array}{l}\text { Outros componentes (argolas, } \\
\text { enfeites, rebites, etc.) }\end{array}$ & Intrínseca & COMP & $\begin{array}{l}\text { Sim: } \mathrm{COMP}=0 \\
\text { Não: } \mathrm{COMP}=1\end{array}$ \\
\hline
\end{tabular}

Fonte: Silva (2017)

Analisando-se as variáveis, pode-se observar que a totalidade das variáveis são categóricas e por isso utilizou-se variáveis dummy. Quando estas variáveis são utilizadas, um dos níveis da variável categórica é retirado e a nova variável é definida como sendo o nível menos $1(n-1)$.
As variáveis Localização de Loja (LOC) e Tipo de Canal de Distribuição (CAN) visam verificar se esses aspectos influenciam os preços dos calçados sociais masculinos e foram desenvolvidas tendo como base Parente (2014), que apresenta a variável Localização Varejista. O mesmo ocorreu com as 
variáveis Região da Cidade de São Paulo (REG) e Classificação dos Distritos Municipais de São Paulo (CLAS), que tem como objetivo avaliar se os preços dos calçados sofrem influência da região em que as lojas se situam e do perfil econômico dos prováveis consumidores. Estas duas últimas variáveis foram criadas de acordo com Parente (2014), que apresenta as Variáveis Ambientais, em conjunto com o Atlas Ambiental da Prefeitura de São Paulo (2016).

A marca é definida como um símbolo capaz de identificar um produto ou serviço de um fornecedor, diferenciando-os dos oferecidos pelos concorrentes da empresa (Kotler e Keller, 2006). As 55 marcas diferentes de calçados sociais masculinos (Companhia Nacional de Calçados - CNS, Fushida, etc.) obtidas pela pesquisa foram classificadas como conhecidas ou pouco conhecidas. A intenção era identificar se há influência da marca no preço do calçado. A variável Número de Parcelas (NPARC), classificada como discreta, e que lista o número de parcelas que cada loja ofertava em cada sapato coletado na amostra, tem como objetivo avaliar se o número de parcelas modifica o preço do calçado.

A variável Agrupamento por Tipo de Calçado (AGRUP) separou os seis modelos estudados em dois grupos por similaridade para verificar se, por grupo, haveria alterações no preço dos calçados. O primeiro grupo foi composto pelos modelos Oxford, Derby, e Brogue, enquanto o segundo grupo foi formado pelos modelos Loafer, Monk e Side Gore. Quanto a origem (ORCAL), os calçados masculinos são fabricados em dois polos no País: Franca (SP) e Serra Gaúcha (RS). Assim, o objetivo dessa variável era verificar se esses polos influenciam o preço do calçado.

A variável Modelo do Calçado Social Masculino (MOD) envolveu os modelos de calçados sociais masculinos e teve como fonte Rocha (2010). Este autor listou como sendo cinco os calçados masculinos (Oxford, Derby, Monk, Loafer e Mocassim). Destes calçados, apenas o mocassim não é utilizado como sapato social. E não listou os modelos Brogue e o Side Gore, que também são modelos muito utilizados pelos consumidores do sexo masculino como sapatos sociais. Por isso, para esta pesquisa, considerou-se que os modelos de calçados sociais masculinos são seis: Oxford, Derby, Loafer, Monk, Brogue e Side Gore.

As variáveis independentes do estudo de Kumar e Deodhar (2014) - como composição do cabedal, cor, textura, estrutura (bico do calçado), laço, superfície e fivela - foram utilizadas neste estudo (Quadros 7):

Quadro 7 - Variáveis independentes utilizadas no estudo de Kumar e Deodhar (2014)

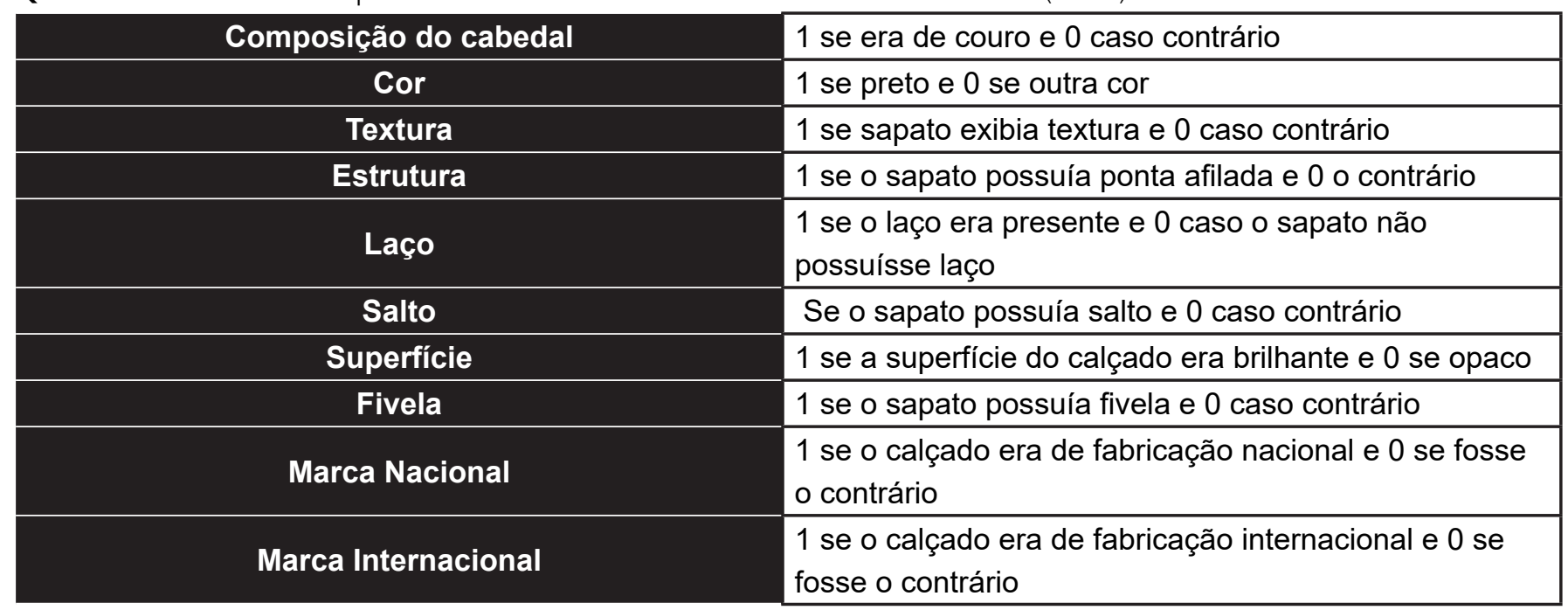

Fonte: Silva (2017) 
Porém várias dessas variáveis foram modificadas após uma revisão na literatura sobre os materiais e componentes utilizados na fabricação de sapatos, com a utilização do livro de Choklat (2012) sobre design de sapatos e de materiais como o dossiê técnico Modelagem Técnica de Calçados, de Schmidt (2007) para se identificar atributos como variações de cor, tipos de couro usados na fabricação, etc. A variável Solado (SOL), composta de três classificações: solado de couro, misto (couro + borracha), e borracha (natural ou sintética); e a variável Outros componentes (COMP) foram variáveis criadas depois de se avaliar os materiais bibliográficos já descritos.

Para determinar o modelo de preços, quatro diferentes modelos (Lin-Lin, Lin-Log, Log-Lin e Log-Log) foram elaborados. Para a escolha das variáveis independentes, dentre os quatro métodos existentes (Enter, Forward Selection, Backward Elimination e Stepwise) optou-se pelo método stepwise, que seleciona as variáveis mais significativas para o modelo, além de ser uma solução para a multicolinearidade. Nos modelos Lin-Lin e Lin-Log foram eliminadas as variáveis laço e agrupamento, por possuírem multicolinearidade elevada (VIF = 21,68 e 21,70, respectivamente).

Para se decidir qual desses modelos tem o melhor R2 ajustado, foi utilizado o critério Linktest. O Linktest é empregado para se avaliar se um modelo está corretamente especificado. Para isso o Linktest cria duas novas variáveis, uma delas denominada "hat" (variável de previsão) e a outra denominada "hatsq", que é o quadrado da variável de previsão. O modelo é refeito com essas duas variáveis independentes e espera-se que a variável "hat" seja significativa, pois se o modelo está corretamente especificado, as previsões ao quadrado não devem ter poder de explicação significativo. Assim, o valor do p-value de hatsq deve ser maior do que 0,05, para que a regressão seja considerada corretamente especificada (FÁVERO, 2015; STATA, 2016). Os modelos e os critérios podem ser vistos na Tabela 2:

Tabela 2 - Sumário de informações para escolha do melhor modelo

\begin{tabular}{lllllll}
\multicolumn{1}{c}{ Model } & $\begin{array}{c}\text { Número de } \\
\text { Variáveis } \\
\text { independentes }\end{array}$ & $\mathbf{R 2}$ & $\mathbf{R 2}$ ajustado & VIF médio & hat & hatsq \\
\hline Lin-Lin & 15 & 0,7880 & 0,7851 & 1,37 & 0,000 & 0,420 \\
\hline Lin-Log & 15 & 0,7925 & 0,7897 & 1,37 & 0,000 & 0,476 \\
\hline Log-Lin & 18 & 0,6945 & 0,6895 & 1,78 & 0,000 & 0,001 \\
\hline Log-Log & 18 & 0,7185 & 0,7139 & 1,56 & 0,000 & 0,000 \\
\hline
\end{tabular}

Fonte: Silva (2017)

Os melhores modelos obtidos, de acordo com o R2 ajustado e Linktest, foram os modelos Lin-Lin e Lin-Log. Quando observados mais cuidadosamente, os dois modelos são praticamente iguais, diferindo apenas na variável "número de parcelas", que no modelo Lin-Log é logaritimizada, enquanto no modelo Lin-Lin essa variável é linear. Optou-se pela utilização da forma funcional linear pela facilidade de compreensão ao permitir que os resultados possam ser observados diretamente, por informarem o valor direto da contribuição do atributo para o preço final do produto (FOUTO; FRANCISCO, 2010). 
Após a escolha da forma funcional calculou-se distância de Cook, que resultou na eliminação de 63 outliers da amostra. Seguiu-se nova regressão que pode ser vista na Equação 7:

Preço do calçado no varejo $=318,5565+$ 9,548842 MOD1 - 18,09449 REG01 - 19,92589 LOC + 10,62437 FIV + 24,01827 COU2 - 44,76934 REG04 - 36,67595 CAN - 63,4011 REG03 47,59493 SURF1 - 39,91071 SOL1 - 38,01952 MARCA - 46,42343 BIC + 13,83376 NPARC - 75,04705 CLAS - 77,83178 SOL2 + 593,923 COU4 (7)

O R2 encontrado foi de 0,7998. O critério Linktest também foi refeito e indicou que a forma funcional continuava apropriada (hat $=0,000$ e hatsq $=0,541)$. O resultado do teste de multicolinearidade (VIF/Tolerance) mostrou um valor aceitável (média de 2,11 - sendo o maior VIF $(4,80)$ encontrado na variável Região da Cidade Central - REG1) O teste Breusch-Pagan indicou a existência de heterocedasticidade ao nível de significância de 5\% (Chi2 = 5,85 e Prob>chi2 > 0,0158). Para correção da heterocedasticiodade utilizou-se o método de Huber-White, com o uso da regressão com erro padrão robusto com um nível de confiança de 95\%. Os resultados estatísticos da Equação 7 após a aplicação da regressão robusta podem ser visualizados na Tabela 3:

Tabela 3 - Estatísticas do modelo após a regressão robusta

\begin{tabular}{cccccc}
$\begin{array}{c}\text { Variável } \\
\text { explicativa }\end{array}$ & Coeficiente & $\begin{array}{c}\text { Lin-Lin } \\
\mathbf{T}\end{array}$ & $\mathbf{P}>\mathbf{| t}$ & $\begin{array}{c}\text { Robust } \\
\mathbf{t}\end{array}$ & $\Delta \% \mathbf{~}$ \\
\hline COU4 & 593,923 & 104,39 & 0,000 & 104,39 & $254,59 \%$ \\
\hline SOL2 & $-77,83178$ & $-22,40$ & 0,000 & $-22,4$ & $-1,19 \%$ \\
\hline CLAS & $-75,04705$ & $-20,53$ & 0,000 & $-20,53$ & $4,48 \%$ \\
\hline NPARC & 13,83376 & 11,69 & 0,000 & 11,69 & $-13,15 \%$ \\
\hline BIC & $-46,42343$ & $-12,42$ & 0,000 & $-12,42$ & $-8,61 \%$ \\
\hline MARCA & $-38,01952$ & $-9,19$ & 0,000 & $-9,19$ & $-9,64 \%$ \\
\hline SOL1 & $-39,91071$ & $-6,95$ & 0,000 & $-6,95$ & $4,20 \%$ \\
\hline SURF1 & $-47,59493$ & $-7,35$ & 0,000 & $-7,35$ & $6,21 \%$ \\
\hline REG03 & $-63,4011$ & $-7,65$ & 0,000 & $-7,65$ & $-18,18 \%$ \\
\hline CAN & $-36,67595$ & $-5,47$ & 0,000 & $-5,47$ & $2,24 \%$ \\
\hline REG04 & $-44,76934$ & $-7,87$ & 0,000 & $-7,87$ & $1,55 \%$ \\
\hline COU2 & 24,01827 & 5,40 & 0,000 & 5,4 & $15,14 \%$ \\
\hline FIV & 10,62437 & 3,24 & 0,001 & 3,24 & $0,00 \%$ \\
\hline LOC & $-19,92589$ & $-2,69$ & 0,007 & $-2,69$ & $-20,18 \%$ \\
\hline REG01 & $-18,09449$ & $-2,31$ & 0,021 & $-2,31$ & $-5,33 \%$ \\
\hline MOD1 & 9,548842 & 2,21 & 0,028 & 2,21 & $-8,30 \%$ \\
\hline Constante & 318,5565 & 32,59 & 0,000 & 32,59 & $-15,17 \%$ \\
\hline Fiva $(2017)$ & & &
\end{tabular}

Fonte: Silva (2017)

A regressão com erros padrão robusta não afeta os resultados do Linktest e mantém os mesmos valores para os coeficientes do modelo original. Mas aumentou a significância para as variáveis
"Cromo Alemão" e "Couro Ovino". Para outras variáveis ocorreu diminuição no valor da estatística t, como na variável "Número de Parcelas", "Região Sul" e "Localização da Loja". A significância 
da constante da regressão também sofreu redução (Tabela 2). O modelo final obtido também atende ao requisito econométrico da normalidade dos resíduos e possui um R2 de $80 \%$, o que indica que o modelo captura boa parte da variação dos preços dos calçados encontrados nesta pesquisa. O modelo também é confiável (estatística $\mathrm{F}$ teve resultado igual a 0,0000) e possui relação linear.

\section{CONSIDERAÇÕES FINAIS}

O estudo teve como objetivo identificar e mensurar os valores dos fatores que afetam o merca- do varejista de calçados no município de São Paulo. Os resultados encontrados são bastante interessantes. A constante do modelo, que é estatisticamente significante na regressão, indica que o preço básico de um calçado masculino deve ser $\mathrm{R} \$ 318,56$. Esse valor de acordo com Kumar e Deodhar (2014) captura a influência de variáveis que não estão incluídas de maneira explícita na equação de preços hedônicos, como, por exemplo, solados como palmilhas confortáveis ou se os sapatos são leves ou pesados. Além disso, foram identificados fatores que impactam positivamente e negativamente o preço dos calçados masculinos no município de São Paulo. Esses resultados podem visualizados na Tabela 4:

Tabela 4 - Fatores que aumentam ou diminuem o preço do calçado social masculino em ordem decrescente

\section{Preço (R\$) Fatores que diminuem o preço}

\begin{tabular}{llll}
77,83 & Solado de borracha & 593,92 & Couro tipo cromo alemão \\
\hline 75,05 & Distrito popular & 24,02 & Couro de carneiro \\
\hline 63,40 & Região Sul da cidade de São Paulo & 13,83 & Número de parcelas \\
\hline 47,59 & Acabamento de superfície & 10,62 & Ausência de fivela \\
\hline 46,42 & Bico quadrado ou afilado & 9,55 & Modelo Side Gore \\
\hline 44,77 & Região Norte da cidade de São & \\
\hline 39,91 & Paulo & \\
\hline 38,02 & Solado misto (borracha + couro) & \\
\hline 36,68 & Marca pouco conhecida & \\
\hline 19,93 & Loja independente & \\
\hline \multirow{2}{*}{18,09} & Loja localizada em shopping & \\
& Região Central da cidade de São & \\
\end{tabular}

Fonte: Silva (2017)

Pode-se constatar que o fator que mais desconta o preço do calçado é a presença do Solado de Borracha (natural ou sintética), que faz com o preço do calçado caia de $\mathrm{R} \$ 318,56$ para $\mathrm{R} \$$ 240,73, uma variação negativa de 24,43\%, possivelmente uma indicação de que os consumidores não estão dispostos a pagar mais por esse tipo de solado. Outro fator que, por sua vez, aumenta o a ser $R \$ 912,48$, o que significa um aumento de $186,43 \%$, é a utilização de Couro tipo Cromo Alemão, que é o couro bovino obtido de gado confinado e de até dois anos de vida curtido com cromo e posteriormente recurtido com tanino vegetal, e preço do sapato, fazendo com que seu valor passe 
que apresenta características intermediárias entre o couro do bezerro recém-nascido e o do gado adulto. Esse é um insumo mais caro para a fabricação de calçados, mas ao mesmo tempo o resultado indica que os consumidores estão dispostos a pagar um prêmio sobre o preço por calçados feitos com esse material, possivelmente por considerarem que um calçado fabricado com esse tipo de material confere maior status.

As variáveis referentes às regiões e distritos da cidade de São Paulo levaram em consideração a renda média domiciliar por distrito que foram obtidas do Atlas Ambiental de São Paulo. Os resultados encontrados para as Regiões Sul, Norte e Central indicam que os calçados vendidos nessas regiões do município de São Paulo são mais baratos, possivelmente em função do menor poder aquisitivo que predomina nessas regiões. O mesmo ocorre em relação à variável classificação dos distritos municipais (CLAS), que indica que quando o distrito é popular, o preço do calçados é descontado em $\mathrm{R} \$ 75,05$.

Outro fator que diminui o preço dos calçados sociais masculinos é se as lojas são de shopping, o que implica em um decréscimo de $\mathrm{R} \$ 19,93$. Caso sejam lojas independentes, o preço será também diminuído em $\mathrm{R} \$ 36,68$. Isso indica que os consumidores esperam encontrar sapatos mais baratos caso as lojas sejam de shopping ou independentes.

Os fatores que aumentam o preço do calçado, ou seja, aqueles pelos quais o consumidor está disposto a pagar um prêmio no preço no mercado paulistano são: couro de carneiro (COU2), ausência de fivela (FIV) e se o modelo do calçado é o Side Gore (MOD1). Todos esses fatores acrescentam aumentam o preço em $\mathrm{R} \$$ 9,55 (modelo Side Gore), $R \$ 10,62$ (ausência de fivela) e $R \$ 24,02$ (caso o sapato seja feito com couro de carneiro). Em relação à fivela, no mercado indiano este item aparece no estudo como sendo um possível símbolo de estilo em sapatos de luxo, fazendo com que os indianos tenham disposição de pagar 29\% a mais por um calçado com esse acessório. No mercado paulistano, o resultado é o inverso: o consumidor masculino aparentemente prefere calçados que não tenham fivela. Uma informação interessante é que se a variável número de parcelas (NPARC) efetivamente mostra que o consumidor está disposto a pagar um acréscimo de $R \$ 13,83$ pela facilidade de poder fazer compras parceladas a prazo. Porém não foi possível determinar efetivamente se há diferenças de prêmio entre os diversos parcelamentos coletados.

Os resultados encontrados possuem algumas similaridades e também algumas diferenças em relação do estudo inicial de Kumar e Deodhar (2014). No caso da variável MARCA os pesquisadores indianos estudaram se a marca do calçado era nacional ou internacional. Como resultado concluíram que os consumidores estão dispostos a pagar um prêmio sobre o preço bastante expressivo - de 120 a $128 \%$ - caso a marca seja reconhecida nacional ou internacionalmente, quando comparada com outras marcas locais. Nesta pesquisa, a marca foi classificada entre conhecida (exemplo a CNS Companhia Nacional de Calçados) e pouco conhecida (como por exemplo a Fushida). Como resultado obteve-se que uma marca pouco conhecida reduz significativamente o preço do calçado aos olhos do consumidor em $\mathrm{R} \$ 38,02$.

No estudo indiano, o formato da ponta do sapato (Bico), que eles denominaram estrutura, foi considerado insignificante para a análise, não pos- 
suindo assim influencia sobre o preço do calçado. Nesta pesquisa a variável bico (BIC) é estatisticamente significativa e diminui o preço do calçado em $R \$ 46,42$, caso o bico seja quadrado ou afilado. Isso sugere que o consumidor brasileiro tem preferência pelos calçados de ponta redonda, em detrimento dos calçados de ponta afiliada ou quadrada.

Em relação ao acabamento de superfície do sapato, o estudo indiano indicou que os homens estavam dispostos a pagar um preço $28 \%$ superior caso o calçado tivesse superfície brilhante. E concluíram que talvez os consumidores vejam valor em comprar sapatos que não necessitem de polimentos frequentes. Já neste estudo, se o acabamento de superfície (SURF) é fosco, o preço do sapato sofre uma redução de $R \$ 47,59$, indicando que no mercado paulistano os homens também têm preferência por sapatos semi-foscos ou de acabamento brilhante. Ou seja, da mesma forma que os indianos, os homens em São Paulo preferem calçados que necessitem de menos polimentos, da mesma forma que os indianos.

Por fim, a variável Solado Misto (borracha + couro) (SOL1) também diminui o preço do calçado em 12,53\%. Em conjunto com a variável Solado de Borracha (SOL2), isso parece indicar que os consumidores preferem que sapatos sociais masculinos tenham solado de couro, ao invés de solado misto ou de borracha.

Os resultados obtidos confirmam a hipótese do estudo de que este método pode ser aplicado em calçados e também evidenciam os preços implícitos das características mais relevantes. O modelo final possui um poder de explicação significativo (R2 de 80\%), sendo resultado de ações que envolveram a eliminação de outliers, exclusão de variáveis para ajustar a multicolinearidade, e emprego de regressão robusta para corrigir a heterocedasticidade. O modelo também possui relação linear, os resíduos da regressão indicam normalidade e o teste de especificação Linktest demonstra que a forma funcional do modelo (linear) está correta.

No âmbito acadêmico a contribuição teórica deste estudo visa somar ao conhecimento do tema. Do ponto de vista gerencial os resultados encontrados podem contribuir para melhora do portfólio de produtos, identificação do preço de entrada de novos produtos, e melhor segmentação de mercado, pois as diferentes características usadas nos sapatos, bem como as regiões em que são comercializadas, como visto neste estudo, impactam positivamente ou negativamente o preço dos sapatos. A análise dos resultados também pode ajudar os gestores a tomarem decisões sobre investimentos de longo prazo ajudando-os na alocação correta dos recursos necessários para a obtenção de determinadas características.

Como limitações da pesquisa, tem-se que o uso da amostragem intencional faz com que os resultados não possam ser generalizados. Outra limitação refere-se ao preço do calçado, que exibiu grande amplitude, variando de $\mathrm{R} \$ 50,00 / p a r$ até $\mathrm{R} \$ 1000,00 /$ par. Neste sentido, sugere-se como um possível estudo retirar os valores extremos da amostra para uma análise baseada mais na média de preços do mercado. Outra possibilidade de pesquisa é fazer um levantamento dos custos e fazer uma análise de preços hedônicos com esses valores, tanto no atacado quanto no varejo, verificando os atributos mais importantes em cada canal de vendas. Também se podem realizar estudos semelhantes ao que foi feito neste trabalho com calçados femininos ou infantis. 


\section{REFERÊNCIAS}

AGUIRRE, A.; FARIA, D. M. C. P. A utilização de "preços hedônicos" na avaliação social de projetos. Revista Brasileira de Economia (RBE), Rio de Janeiro, v. 51, n. 3, p. 391-411, 1997.

ALVES, V. C.; BARBOSA, A. S.. Práticas de gestão ambiental das indústrias coureiras de Franca-SP. Gestão \& Produção, São Carlos, v. 20, n. 4, p. 883-898, 2013.

ASSOCIAÇÃO BRASILEIRA DE EMPRESAS DE COMPONENTES PARA COURO, CALÇADOS E ARTEFATOS (ASSINTECAL). Estudo dos polos calçadistas brasileiros, 2011.

ATLAS AMBIENTAL DA PREFEITURA DE SÃO PAULO. Renda média domiciliar por distrito municipal da cidade de São Paulo. Disponível em: http://atlasambiental.prefeitura.sp.gov.br/conteudo/socioeconomia/ socioeco_04.jpg. Acesso em: 29 abr. 2016.

BAGGIO, J.; CATAPAN, A.; MEZA, M. L. F. G. Formação dos preços hedônicos de imóveis residenciais: um estudo de caso a partir das equações de preços hedônicos. In: XVIII Seminários em Administração (SEMEAD) FEA-USP. São Paulo: 2015.

BIMBATTI, M. L. Como enfrentar o "fenômeno China" na produção de calçados: proposta de estratégia competitiva para a indústria. 1994. 257 p. Tese de Doutorado (Doutorado em Engenharia de Produção). Escola Politécnica da Universidade de São Paulo (EPUSP).

BRACHINGER, H. W. Statistical theory of hedonic price indexes, DQE Working Paper $N^{\circ} 1$, University of Friburg, Friburg, Switzerland, 2002.
BUONANNO, P.; MONTOLIO, D.; RAYA-VILCHEZ, J.M. Housing prices and crime perception. Empirical Economics, v. 45, p. 305 - 321, 2013

CAMPOS, E. M. G.; CIRINO, J. F.; ANDRADE, D. $C$. Modelo de regressão para estimar o diferencial de preços das terras agrícolas com e sem erosão em lagoa dourada (MG) pelo método dos preços hedônicos. In: Encontro da Sociedade Brasileira de Pesquisa Operacional (SBPO), 36., 2004. São João del Rei, MG, 2004. Anais... São João del Rei, MG, 2004.

CARDOSO , E. E.; GOMES, A.; LÍRIO, V. S.; LEITE, E. R.; NETO, J. F. T.; COUTINHO, M. E.; CRUZ, G. M.; PACHECO, M. A.; VELLY, M. L. M. Análise da cadeia produtiva de peles e couros no Brasil. EMBRAPA, Comunicado Técnico, Campo Grande, n. 68, p. 1- 4, 2001.

CHAU, K. W.; CHIN, T. L. A critical review of literature on the hedonic price model. International Journal for Housing Science and Its Applications, v. 27, n. 2, p. 145-165, 2003.

CHOKLAT, A. Design de sapatos. São Paulo: Editora Senac São Paulo, 2012.

CONSTANZI, R. N. Distribuição espacial da indústria de calçados no Brasil no século XX. 219 p. Dissertação (Mestrado em Administração) - Faculdade de Economia e Administração da Universidade de São Paulo. São Paulo, 1999.

COSTA, A. B. Modernização e competitividade da indústria de calçados brasileira. Rio de Janeiro: s.ed., 1993. 


\section{REFERÊNCIAS}

COSTA, A. B.. A trajetória competitiva da indústria de calçados do Vale dos Sinos. In: COSTA, A. B.; PASSOS, M. C. (Org.). A indústria calçadista no Rio Grande do Sul. São Leopoldo: Unisinos, 2004.

DRESDNER, J.; LAVIN, F.; AGUILAR, R. The value of air quality and crime in Chile: a hedonic wage approach. Enviroment and Development Economics Review, v. 16, n. 3, p. 329-355, 2011.

DUTTON, J. M.; WARD, C. E. LUSK, J. L. Implicit value of retail beef brands and retail meat product attributes. Proceedings of the NCCC-134 Conference on Applied Commodity Price Analysis, Forecasting, and Market Risk Management. Chicago, 2007.

FÁVERO, L.P.L. Análise de dados: modelos de regressão com Excel®, Stata $®$ e SPSS $®$. Rio de Janeiro: Elsevier, 2015.

FENSTERSEIFER, J. E. (org.). O complexo calçadista em perspectiva: tecnologia e competitividade - um estudo sobre a competitividade da indústria calçadista sob a ótica da tecnologia. Porto Alegre: Ortiz, 1995.

FOUTO, N. M. M. D.; ANGELO, C. F.; LUPPE, M. R. A five-year hedonic price breakdown for desktop personal computer attributes in Brazil. BAR, Braz. Adm. Rev. Curitiba, v. 6, n. 3, p. 173-186, 2009.

FOUTO, N. M. M. D.; FRANCISCO, E. S. Valuation of quality attributes in the price of new economy cars. Revista de Gestão da USP (REGE). v. 18, n. 2, p. 245-258, 2011.
GRANERO, A. E. A linguagem da marcas de calçados da moda: um enfoque publicitário. 258 p, 2006. Tese de Doutorado (Doutorado em Comunicação) - Escola de Comunicação e Artes da Universidade de São Paulo (ECA-USP), São Paulo.

GRILICHES, Z. Hedonic price indexes for automobiles: an econometric analysis of quality change. In National Bureau of Economic Research (Ed.). The Price Statistics of the Federal Government. v. 73, p. 137-196, 1961. New York: Columbia University Press.

GUJARATI, D. N. Econometria básica. 4. ed. Rio de Janeiro: Elsevier, 2006.

LI, H.; WEI, Y.D.; YU, Z.; TIAN, G. Amenity, accessibility and housing values in metropolitan USA: A study of Salt Lake County, Utah. In Cities, v. 59, p. 113-125, 2016.

INSTITUTO DE ESTUDOS DE MARKETING INDUSTRIAL (IEMI). Relatório setorial da indústria de calçados do Brasil, 2014.

INSTITUTO DE ESTUDOS DE MARKETING INDUSTRIAL (IEMI). Relatório setorial da indústria de calçados do Brasil, 2015.

JABBAR, M. A. Buyer preferences for sheep and goats in southern Nigeria: a hedonic price analysis. Agricultural Economics, v. 18, p. 21-30, 1998.

KARIPIDIS, P. TSAKIRIDOU, E.; TABAKIS, N.; KONSTANTINOS, M. Hedonic analysis of retail egg price. J Foods Dist Res, v.36, n. 3, p. 68-73, 2005. 


\section{REFERÊNCIAS}

KEMPF, S. Development of hedonic office rental indices: examples for german metropolitan areas. In Rottke, N. B.; Mutl, J. (orgs) Essays in real estate research, Band 5, 2015.

KOKOSKI, M.; WAEHRER, K.; ROZAKLIS, P. Using Hedonic Methods for Quality Adjustment in the CPI: The Consumer Audio Products Component, BLS Working Papers, n. 344, 2001.

KUMAR, V.; DEODHAR, S. Y. From well-heeled to tip-toed, shoe-shine to shoe-lace: valuing product differentiation in men's formal footwear. Working Paper. Indian Institute of Management, India, 2014.

LANCASTER, K. A new approach to consumer theory. Journal of Political Economy, v. 74, n. 2, p. 132-157, 1966.

MACHADO NETO, A. J. Os determinantes do comportamento exportador da indústria calçadista francana. 279 p, 2006. Tese de Doutorado (Doutorado em Administração) - Faculdade de Economia, Administração e Contabilidade da Universidade de São Paulo - FEA-USP - São Paulo, 1996.

MENESES, R.; BRITO, P. Q.; GOMES, P.C. From offshore-provider to brand creator: fsQCA of footwear industry. Journal of Business Research, v. 69, 11 ed., p. 5540-5546, 2016.

MENEZES, M.S; PASCHOARELLI, L.C.(orgs). Design e planejamento: aspectos tecnológicos [online]. São Paulo: Editora UNESP; São Paulo: Cultura Acadêmica, 277 p, 2009.
MUNÕZ, R. R.; MOYA, M. L.; GIL, J. M. Market values of olive oil attributes in Chile: a hedonic price function. British Food Journal, vol. 117, n.1, pp. 358-370, 2015.

ORREGO, M. J.E; DEFRANCESCO, E.; GENNARI, A. The wine hedonic price models in the "Old and New World": state of the art. Rev. FCA UNCuyo, v. 44, n. 1, p. 205-220, 2012.

PARENTE, J. Varejo - Gestão e Estratégia. 2. ed. São Paulo: Atlas, 2014.

PRADIER, PC.; GARDIER, F.; GREFFE, X.; MENDOZA, I. M. Jounal of Cultural Economics, v. 40, p. 453-485, 2016.

RAO, $V$. The rising price of husbands: a hedonic analysis of dowry increases in rural India. Journal of Political Economy, 65, pp. 23-28, 1993.

ROHEIM, C.A.; GARDINER, J.; ASCHE, F. Value of brands and other attributes: hedonic analysis of retail frozen fish in the UK. Marine Resource Economics, v. 22, p. 239-253, 2007.

ROSEN, S. Hedonic prices and implicit markets: product differentiation in pure competition. Journal of Political Economy, v. 82, n. 1, pp.34-55, 1974.

SILVA, A. M. Precificação dos atributos dos calçados sociais masculinos na cidade de São Paulo: uma análise de preços hedônicos. 2017. Tese (Doutorado em Administração) - Faculdade de Economia, Administração e Contabilidade, Universidade de São Paulo, São Paulo, 2017. 


\section{REFERÊNCIAS}

SCHMIDT, M. R. Modelagem técnica de calçados. Porto Alegre: Centro Tecnológico do Calçado SENAI, 2007.

STANLEY, L.R.; TSCHIRHART. J. Hedonic prices for a non-durable good: the case of breakfast cereals. The Review of Economics and Statistics. v. 73, n. 3, p. 537-541, 1991.

STATA. Linktest, 2016.

TRIPLETT, J. E. Automobiles and hedonic quality measurement. Journal of Political Economy. v. 77, no 3, pp. 408 - 417, 1969.

VERDU-JOVER, A.; GOMEZ-GRAS, J.; MARTINEZ-MATEO, J. Value creation through production offshore-inshore strategies in a footwear industry cluster: a coevolutionary perspective. International Business Review, VOL. 21, 3 ed., p.342-356, 2012.

VIANA, F. E; ROCHA, R. E. V. A indústria de calçados no Nordeste: características, desafios e oportunidades. Fortaleza: Banco Nordeste do Brasil, 2006.

YANG, Y; MUELLER, N. J.; CROES, R. R. Market accessibility and hotel prices in the Caribbean: The moderating effect of quality-signaling factors. Tourism Management, v. 56, p. 40-51, 2016.

XIAO, Y. Urban Morphology and Housing Market. Springer Geography Series. Tongji Univesity Press and Springer Nature Singapure Pte Ltd., 2017. 